\title{
Evidence-based comparison of molecular-genexpert and microscopic method in the diagnosis of mycobacterium tuberculosis among subjects in the limited resource setting of Niger delta
}

\begin{abstract}
Increasingly, pragmatic disease diagnosis is important for treatment, as well as disease prevention and control strategy, thus timely and accurate diagnostic outcome, may help to prevent or reduce the negative consequences of the disease in the body and the population at large, even as well as reduce mortality index cases in the affected community or region. This study was carried out to determine an effective method for the detection of Mycobacterium tuberculosis (MTB) among suspected tuberculosis patients visiting the University of Port Harcourt Teaching Hospital (UPTH) and the Braithwaite Memorial Specialist Hospital (BMSH), all in Port Harcourt, Nigeria. A total of 286 and 269 sputum samples were collected from the UPTH and BMSH respectively, making a total of 555 samples. The two methods explored were ZN -Microscopy and the GeneXpert assay method. Using GeneXpert (Modules Thermal and Optical System Cepheid, Serial Number, 805757 , USA) $26.2 \%$ and $30.9 \%$ of the samples were positive for MTB in UPTH and BMSH, respectively. While microcopy detected $15.4 \%$ and $29.4 \%$ MTB samples from UPTH and BMSH respectively. Four (4) positive cases were detected by microscopy which was not detected by GeneXpert. Nonetheless, in overall, the sensitivity of GeneXpert assay was higher than that of microscopy. It is therefore strongly believed that the combination of the conventional method and the molecular assay or improvement of the molecular method may enhance detection of MTB in clinical samples especially in endemic regions where majority of the populace may be at risk due to seemingly poor living condition, poverty and limited access to prompt health education and robust health facility.
\end{abstract}

Keywords: Mycobacterium tuberculosis, genexpert, microscopy, comparison, Niger delta, diagnosis, disease
Volume 6 Issue 2 - 2018

\author{
Wokem Gloria Ngozika,' Azuonwu Obioma,' \\ Josiah Nkereawaji Appollus ${ }^{2}$ \\ 'Department of Medical Laboratory Science, Rivers State \\ University of Science and Technology, Nigeria \\ ${ }^{2}$ Department of Medical Microbiology, University of Port \\ Harcourt Teaching Hospital Choba East-West Road, Nigeria
}

Correspondence: Azuonwu Obioma, Department of Medical Laboratory Science, Rivers State University of Science and Technology Nkpolu Orowhorukwo, Port Harcourt, Nigeria, Te 234 803551968,Email bimajacobs@yahoo.co.uk

Received: January 30, 2018 | Published: March 23, 2018

\section{Introduction}

At of 2014, tuberculosis (TB) was termed the world's deadliest communicable disease and is caused by Mycobacterium tuberculosis (MTB). ${ }^{1}$ The disease is transmitted from one infected person to a healthy individual(s) through air droplets or inhalation of aerosols from an infected subject. ${ }^{2}$ Worldwide, the tuberculosis burden is high with an estimation of 8.7 million new cases in 2012. ${ }^{3}$ Nevertheless, $80 \%$ of this was from 22 countries, the majority of which were diagnosed as smear-negative pulmonary tuberculosis (SN-PTB). These countries rely on the conventional method of detection of MTB using smear microscopy whose reliability ranges from $20 \%$ to $60 \%{ }^{4,5}$ Nonetheless, PTB is a popular opportunistic infection in Sub-Saharan Africa (Nigeria inclusive) where there is a high prevalence of HIV; usually persons with TB/HIV co-infection present with SN-PTB. This is as a result of the poorly formed lung granulomas/cavities in TB patients which leads to a reduced quantity of MTB in the lesions and subsequently cause difficulties during diagnosis of the infection. ${ }^{6,7}$

Overtime, early diagnosis has proved to be important in the early management and possible cure of the disease and its clinical complications. Tuberculosis is one of such diseases that require careful and accurate diagnosis outcome. It is caused by the bacteriumMycobacterium tuberculosis (MTB). Three methods are most often used in the diagnosis of TB and these are microscopy, culture and GeneXpert. Of these, microscopy is not as sensitive and specific as GeneXpert which is described as a molecular technique for MTB detection. ${ }^{8}$ However, in most nations, the conventional microscopy, culture and drug susceptibility testing (DST) method is used in TB diagnosis. ${ }^{9}$ There is however, a possibility of a misdiagnosis leading to a false positive or false negative result in cases where culture is done. Furthermore, culture is time consuming, as it requires about 2-6 weeks to get a result and also requires technical expertise $(1,6,7)$. In $2014,68.1 \%$ of the PTB cases recorded in Italy were smear-negative. ${ }^{10}$ The WHO has thus approved the GeneXpert assay for the diagnosis of $\mathrm{TB}$, as it not only detects MTB, but also detects rifampicin resistance strains, and finally it is able to produce results within 2 hours. ${ }^{11}$ This is, therefore, commendable, as it tends to promote health outcome of massive benefit to the supposedly patients.

The choice of GeneXpert as a method of MTB detection was preceded by developments in the area of molecular biology and diagnostics approach, which is currently playing a major role in the swift dynamism of the diagnosis and response to TB health issues 
across the globe. ${ }^{9}$ These advances including those in vaccines and drugs have enhanced the worldwide hope for TB control and prevention. ${ }^{12}$ Molecular methods for the detection of TB are INNO LIPA Rif.TB (Innogenetics, Ghent, Belgium) and GeneType MTBDRplus (Hain Lifescience $\mathrm{GmbH}$, Nehren, Germany) which are line probe assays and GeneXpert MTB/RIF (Cepheid, Sunnyvale, USA) which is a real-time polymerase chain reaction (PCR). ${ }^{13}$

GeneXpert assay involves lysis of the bacteria, extraction of DNA, amplification and amplicon detection. ${ }^{14}$ Its advantages are that it is less complex, requires less time, has high specificity and sensitivity and requires less safety concerns for whoever that will be handling the samples. ${ }^{15-19}$ Nonetheless, this study was carried out to determine which method of assay is best and more sensitive in the detection of MTB in sputum samples from two major health facilities in Port Harcourt, using GeneXpert assay and microscopy. It is strongly believed that data generated would help to improve prompt and precise diagnostic outcome of the disease burden, and thus in the end, patients care and overall case management strategy would be strongly underpinned, especially in our remote communities in Niger Delta where access to functional health care Centre remains a massive challenge.

\section{Methodology}

\section{Sampling area}

This study was carried out in Braithwaite Memorial Specialist Hospital and the University of Port Harcourt Teaching Hospital, which are two hospitals in the state with a GeneXpert Machine. BMSH is located in the Government Residential Area (GRA), Port Harcourt; latitude $12.9416^{\circ} \mathrm{N}$ and $77.5669^{\circ} \mathrm{E}$. It is a state government owned hospital operated by the Rivers State Hospitals Management Board and named after Eldred Curwen Braithwaite, a British surgeon and pioneer surgeon in Rivers State. When it was established in 1925 as Braithwaite Memorial Hospital (BMH), it served as a medical facility for senior civil servants. Later, it became a general hospital but has now become a Specialist Health Institution. It has 731 medical staff and 375 licensed beds.

The UPTH is a federal government owned hospital located in East West Road, Port Harcourt; latitude $4.8996^{\circ} \mathrm{N}$ and $6.9286^{\circ} \mathrm{E}$. It is a major tertiary-care teaching and research health facility in the Niger Delta region of Nigeria established in 1980 but officially commissioned in 1985 . It presently has a 500-bed capacity. The city where both hospitals are located is the largest in Rivers State. The State has 23 Local Government Areas of which Port Harcourt Local Government is one.

\section{Study population}

The city is home to persons from different tribes of the country as well as foreigners who indulge in oil exploration. The dwellers are predominantly civil servants while some indulge in trading, farming and fishing. Suspected PTB patients were used for the study after ethical approval from the State Ministry of health as well as the UPTH had been obtained and the consent of the patients had been sought and obtained after informing them on the reason and relevance of the study. The patients who provided their sputum in sterile sample containers were the test population while non-TB subjects who had tested negative to MTB infection were the control population.

\section{Ethical clearance and subject consent}

Ethical clearance was obtained from the UPTH and the Rivers State Ministry of Health following due process. Informed consent of the patients was also obtained and the confidentiality of the results assured before their sputa was collected. Confidentiality of results was ensured by assigned numbers to samples collected as codes. The study was carried out between January 2016 and September 2016. All willing suspected PTB patients regardless of their sex and age were included in the study through convenience sampling design. Health workers with TB symptoms were also included. These included those with prior PTB history, and those with signs and symptoms of PTB and/or chest $\mathrm{x}$-ray suggestive of PTB from the Directly Observed Therapy Short Course (DOTS) clinic.

\section{Sample collection}

Minimum sputum volume of $2 \mathrm{ml}$ was collected from each patient by deep cough method in the morning of patient's enrollment. An early morning sputum was collected from each patient and tested using the microscopy technique and GeneXpert assay. Each sample was assigned a unique identification number, with the date, age and sex of the patient recorded accordingly. Well-structured questionnaires were given to each subject where the subjects provided demographic information as well as stated their reason for testing for TB. The questionnaires were completed by the patients and returned for analysis.

\section{Experimental analysis \\ GeneXpert sample assay}

This assay is made up of a one-time use multi-chambered plastic cartridge, filled with liquid buffer and lyophilized reagent beads needed for processing of the sample, DNA extraction and heminested real-time Polymerase Chain Reaction (PCR). The cartridge contained lyophilized Bacillus globigii spores that served as internal sample processing and PCR control. The collected sputa were treated with $\mathrm{NaOH}$ and a sample reagent (SR) containing isopropanol. In a ratio of $2: 1$, the SR was added to the sputum and incubated at room temperature for 15 minutes. The mixture was then transferred to the cartridge, the cartridge put into the GeneXpert instrument and an automated process completed the remaining steps. During the sample processing step, the $B$. globigii spores are resuspended automatically and processed during the sample processing step and the resulting DNA amplified during the PCR step. Amplification increases the amount of DNA molecules so as to enable easy detection of the MTB genomeThe standard user interface indicated the absence or presence of MTB and a semi quantitative estimate of the concentration of MTB (very low, low, medium and high). Negative assays were reported as invalid.

\section{Microscopy analysis}

Detection of MTB using microscopy is done by first staining the sputum sample using the Ziehl-Neelsen $(\mathrm{Z}-\mathrm{N})$ stain before viewing under the microscope. This staining method is based on the fact that the bacterium is an acid-fast one. A smear of the sputum is made on a clean dry slide and fixed. It is then covered with carbol fuchsin for 3 minutes; heated, rinsed with running water and decolourized with acid-alcohol for 5 seconds. It is then flooded with methylene blue 
(counterstain) for 30 seconds, rinsed with running water and allowed to air dry, it was viewed under the microscope using the $\mathrm{x} 10$ to focus and X100 oil immersion lens for identification. The bacterium usually appears as a red beaded rod; 0.2-0.5 $\mu \mathrm{m}$ wide and 2-4 $\mu \mathrm{m}$ long.

\section{Results}

The 555 sputum samples collected from the two hospitals were assayed for the presence of MTB using both microscopy and GeneXpert assay method. Nevertheless, GeneXpert assay was able to detect $26.2 \%$ and $30.9 \%$ MTB positive samples in the UPTH and BMSH samples respectively, while microscopy detected $15.4 \%$ and 29.4\% MTB positive samples respectively. Among the seven age groups investigated, ages 30-39 had a higher sample size of 171 and next to it were ages 40-49 while persons less than 10 years had lowest sample size of 21. GeneXpert identified 33.3\% MTB samples among 30-39 age groups while it identified 9.5\% MTB positive samples among $\leq 10$ age group. Microscopy identified $25.1 \%$ and $4.8 \%$ of age group 30-39 and $\leq 10$ respectively. Among males $34.2 \%$ and 28.1 was detected using GeneXpert and microscopy, respectively. Among females, $22.1 \%$ and $15.6 \%$ MTB positive samples was detected using GeneXpert and microscopy, respectively (Figure $1 \& 2$ ) (Table 1).

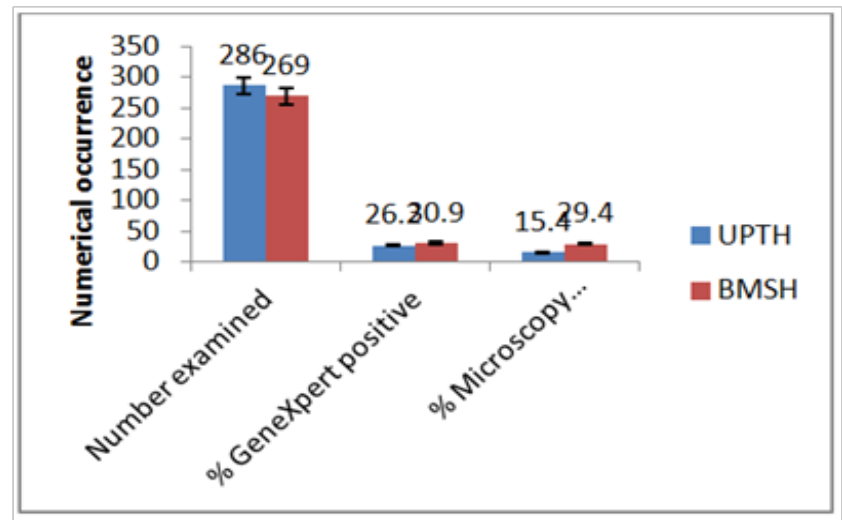

Figure I Percentage detection of MTB using GenXpert and Microscopy

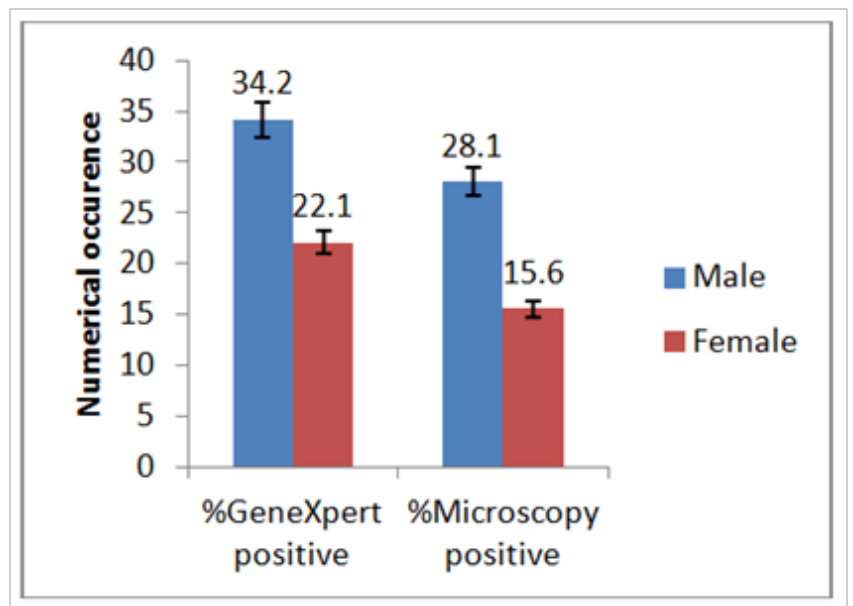

Figure 2 Percentage detection of both methods based on gender
Table I Percentage detection of MTB by both methods based on age

\begin{tabular}{llll}
\hline Age group & $\begin{array}{l}\text { Number } \\
\text { of samples }\end{array}$ & $\begin{array}{l}\text { \%GeneXpert } \\
\text { positive }\end{array}$ & $\begin{array}{l}\text { \% Microscopy } \\
\text { positive }\end{array}$ \\
\hline$\leq 10$ & 21 & 9.5 & 4.8 \\
$19-$ Nov & 45 & 17.8 & 15.6 \\
$20-29$ & 85 & 32.9 & 24.7 \\
$30-39$ & 171 & 33.3 & 25.1 \\
$40-49$ & 122 & 29.5 & 24.6 \\
$50-59$ & 65 & 23.1 & 18.5 \\
$\geq 60$ & 44 & 26.1 & 19.6 \\
\hline
\end{tabular}

\section{Discussion}

Diagnosis of TB for several years had been through microscopy, however in recent times due to developments in molecular biology, GeneXpert assay has been used in the detection of MTB. Previous studies have, however, shown that the conventional methods for the detection of MTB in sputa have low sensitivity. ${ }^{7}$ Although, research has proved that GeneXpert provides quick results in MTB detection, the extraction procedures as well as PCR inhibitors present in some of the samples serve as limitations to its use. ${ }^{9}$ In South Africa a diagnostic sub-study of a tuberculosis prevalence survey was conducted in gold mining companies. Sputum samples were collected and tested using microscopy, liquid culture and GeneXpert. Out of 6893 participants 187 (2.7\%) were positive for Mycobacterium tuberculosis in culture, $144(2.1 \%)$ were positive by GeneXpert and $91(1.3 \%)$ were positive for acid fast bacilli by microscopy. Sensitivity, specificity, Positive predictive value (PV) and negative predictive value (NPV) for detection of Mycobacterium tuberculosis by GeneXpert were $62.6 \%$, $99.6 \%, 81.3 \%$, and 98.9; however, agreement between GeneXpert and culture were $98.5 \%$. Sensitivity of microscopy was $17.6 \%$. When individuals with a history of tuberculosis treatment were excluded from the analysis, GeneXpert specificity was $99.8 \%$ and positive predictive value (PPV) was $90.6 \%$ for detection of Mycobacterium tuberculosis. ${ }^{20}$

A multi-central study was conducted in Peru, Azerbaijan, South Africa, and India to assess the performance of GeneXpert assay in 730 patients with suspected pulmonary tuberculosis. Among culture-positive patients, a single, direct MTB/RIF (Mycobacterium tuberculosis/Rifampicin resistance) test identified 551 of 561 patients with smear-positive tuberculosis $(98.2 \%)$ and 124 of 171 with smearnegative tuberculosis $(72.5 \%)$. The test was specific in 604 of 609 patients without TB (99.2\%). Among patients with smear-negative, culture-positive tuberculosis, the addition of a second MTB/RIF test increased sensitivity by $12.6 \%$ and a third by 5.1 percentage points, to a total of $90.2 \%$. As compared with phenotypic drug-susceptibility testing, MTB/RIF testing correctly identified 200 of 205 patients $(97.6 \%)$ with rifampicin-resistant bacteria and 504 of $514(98.1 \%)$ with rifampicin-sensitive bacteria. Sequencing resolved all but two cases in favor of the GeneXpert assay. ${ }^{21}$ Nonetheless, an assessment by WHO Nigeria has shown that GeneXpert technology provides an opportunity for setting up routine drug resistance surveillance system in the country. Programme implementation data from 22 GeneXpert laboratories for period between September 2011 and December 2013 have already been analyzed by Mustapha et al. (2014) to evaluate 
outcomes and identify challenges and opportunities for strengthening tuberculosis detection in Nigeria. ${ }^{22}$

Nevertheless, from their analysis, a total of 12,249 patients received a single GeneXpert test at 10 secondary, 10 tertiary and 2 private health facilities over 25 months. The tests were valid (ability to detect the presence or absence MTB) in 10,948 patients, and 3669/10948 (33.5\%) were positive for Mycobacterium tuberculosis. In 815/3669 (22.2\%) of the Mycobacterium tuberculosis cases, the bacteria were resistant to rifampicin. Rifampicin resistance was indeterminate in 509/12249 $(4.2 \%)$ while the test failed in 792/12249 (6.5\%). The conclusion was that the GeneXpert MTB/RIF programme implemented in Nigeria targets specific risk groups with a high number of cases detected. ${ }^{22}$ Furthermore, in Abeokuta, Nigeria, Oluwaseun (2012) collected a total of five hundred and four (504) sputum samples by deep cough from TB suspects who registered for the first time at public health centres in Abeokuta. The researchers examined the sputum samples using the Zeihl-Neelsen staining method and GeneXpert assay and compared their results to composite reference standard Lowenstein Jensen culture (LJ) culture. The GeneXpert assay accurately detected 310 previously undiagnosed TB cases, resulting in sensitivity, specificity, positive predictive value and negative predictive value of 98.4\% (95\% CI 97.5-99.4\%), 95.9\% (95\% CI 94.8-98.5\%), 98.8\% (95\% CI 92.6-99.2\%) and 94.0\% (95\% CI 88.5-97.2\%) respectively. For rifampicin resistance detection, sensitivity of GeneXpert was 97.2\% (95\% CI 96.8-98.7\%); Specificity was 99.6\% (95\% CI 92.699.8\%); positive predictive value was $95.2 \%$ (95\%CI 92.6-97.8\%) and negative predictive value was $99.8 \%$ (95\% CI 88.5-99.9\%). Though the case of rifampicin resistance strain was not considered in this present work, the essence of mentioning it is to provide an evidencebased analysis of the sensitivity and specificity of GenXpert method when compared side by side with other methods such as Microscopy. Nevertheless, their conclusion was that GeneXpert performed well for TB diagnosis and rifampicin (RIF) resistance and outperformed smear microscopy. ${ }^{23}$ This study however showed that GeneXpert was much more effective in the detection of MTB when compared with microscopy.

This present study recorded a $28.5 \%$ MTB detection by GeneXpert compared with the $22.2 \%$ detected by microscopy. This is in consonance with the study by Zeka et al. and Ndubuisi et al who recorded $20.2 \%$ and $25.9 \%$ MTB prevalence using GeneXpert assay. ${ }^{24,25}$ Hosne et al. however recorded a $67 \%$ rate in Congo which is more than that recorded in this study. ${ }^{26}$ In contrast to these, Haider et al. recorded a $6.4 \%$ MTB detection rate using GeneXpert which is much lower than that observed in this study. ${ }^{27}$ However, the reasons for above variations is not very clear per se, although, this could be linked to difference in sample size, experimental design strategy and level of expertise and mastery of skills deployed during the sample collection and laboratory practical work outcome during experimental. Nevertheless, it will be evident to state very clearly that four (4) cases of positive cases identified by Microscopy in this study, was reported as negative by GeneXpert; however, the reason is still obscure. This could possibly be aligned to incorrect operation or improper calibration of the machine before use However; all the positives samples detected by GeneXpert were also detected by microscopy. However the importance of enhancing the diagnostic capabilities in our local communities cannot be over- emphasis given the visible absence of functional health care facilities and critical lack of trained and seasoned expertise in this direction to generate much needed diagnostic information's that would obviously enhance the welfare and quick recovery of patients in good time. ${ }^{28,29}$

\section{Conclusion}

Several studies on the use of GeneXpert assay for MTB detection have been done by various researchers, to determine the efficacy of this method. However, this present evidence based study has further demonstrated that GeneXpert is more robust in terms of specificity and sensitivity in the prompt diagnosis of Mycobacterium tuberculosis, especially in endemic regions. But one critical setback that will promote non-accessibility of this method is its cost, especially in resource-limited settings like in the Niger Delta communities and Africa in general, where poverty remains a palpable challenge among citizens and health care providers. Nonetheless, it is strongly recommended that the use of both methods in synergy will certainly improve patients' outcome, even as we will continue to emphasize on the importance of the microscopy method especially in the remote Health Care Centre, in our villages, given its ability to detect MTB in some samples in which GeneXpert was unable to, as well as its relative cost advantage over GeneXpert methods.

\section{Acknowledgements}

The authors are profoundly grateful to all the subjects who willingly participated in the study, even as we are also thankful to all the laboratory staff of both health facilities for their outstanding support during the experimental. We are also grateful to Dr Azuonwu, Goodluck and Miss Chikanka Testimony Azuonwu for taking massive time, out of her tight busy schedule to typeset the manuscript to logical conclusion. We are also indebted to Joy Obi- Azuonwu and Enwereji Hope for their prayers and support.

\section{Conflict of interest}

The authors declare no conflict of interest.

\section{References}

1. World Health Organization. Global tuberculosis report 2014. Switzerland; 2014. $171 \mathrm{p}$.

2. http://www.who.int/mediacentre/factsheets/fs104/en/

3. World Health Organization. Global tuberculosis report 2012 Switzerland; 2012. 100 p.

4. Steingart KR, Ng V, Henry M, et al. Sputum processing methods to improve the sensitivity of smear microscopy for tuberculosis: a systematic review. Lancet Infect Dis. 2006;6(10):664-674.

5. Steingart KR, Henry M, Ng V, et al. Fluorescence versus conventional sputum smear microscopy for tuberculosis: a systematic review. Lancet Infect Dis. 2006;6(9):570-581.

6. Evans CA. GeneXpert-a game changer for tuberculosis control? PLOS MED. 2011;8(7):e1001064.

7. Centers for Disease Control and Prevention. Updated guidelines for the use of nucleic acid amplification tests in the diagnosis of tuberculosis. MMWR Morb Mortal Wkly Rep. 2009;58(1):7-10.

8. Azuonwu O, Ihua N, Koomasiruchi W. Molecular Detection of Mycobacterium tuberculosis (MTB) and Rifampicin Resistant Strain among Subjects Accessing Health Care at Federal Medical Centre, 
Yenegoa, Bayelsa State; Nigeria. Transl Biomed. 2017;8(3):120.

9. Bajrami R, Mulliqi G, Kurti A, et al. Comparison of GeneXpert MTB/RIF and conventional methods for the diagnosis of tuberculosis in Kosovo. The Journal of Infection in Developing Countries. 2016;10(4):418-422.

10. World Health Organization. Antimicrobial resistance: global report on surveillance 2014. Geneva, Switzerland; 2014. 256 p.

11. http://www.who.int/tb/publications/ISTC_3rdEd.pdf

12. Raviglione M, Marais B, Floyd K, et al. Scaling up interventions to achieve global tuberculosis control: progress and new developments. Lancet. 2012;379(9829):1902-1913.

13. Pai M, Minion J, Sohn H, et al. Novel and improved technologies for tuberculosis diagnosis: progress and challenges. Clin Chest Med. 2009;30(4):701-716

14. Lawn SD, Nicol MP. Xpert ${ }^{\circledR}$ MTB/RIF assay: development, evaluation and implementation of a new rapid molecular diagnostic for tuberculosis and rifampicin resistance. Future Microbiol. 2011;6(9):1067-1082.

15. Boehme CC, Nabeta P, Hillemann D, et al. Rapid molecular detection of tuberculosis and rifampin resistance. New Engl J Med. 2010;363(11):1005-1015.

16. Boehme CC, Nicol MP, Nabeta P, et al. Feasibility, diagnostic accuracy, and effectiveness of decentralized use of the Xpert MTB/RIF test for diagnosis of tuberculosis and multidrug resistance: a multicentre implementation study. Lancet. 2011;377(9776):1495-1505.

17. Helb D, Jones M, Story E, et al. Rapid detection of Mycobacterium tuberculosis and rifampin resistance by use of on-demand, near-patient technology. J Clin Microbiol. 2010;48(1):229-237.

18. Zeka AN, Tasbakan S, Cavusoglu C. Evaluation of the GeneXpert MTB/RIF assay for rapid diagnosis of tuberculosis and detection of rifampin resistance in pulmonary and extrapulmonary specimens. $J$ Clin Microbiol. 2011;49(12):4138-4141.

19. Marlowe EM, Novak-Weekley SM, Cumpio J, et al. Evaluation of the Cepheid Xpert MTB/RIF assay for direct detection of Mycobacterium tuberculosis complex in respiratory specimens. J Clin Microbiol. 2011;49(4):1621-1623.

20. Dorman SE, Chihota VN, Lewis JJ, et al. Performance Characteristics of the Cepheid Xpert MTB/RIF test in a Tuberculosis Prevalence Survey.
Public Library of Science. 2012;7(8):433-437.

21. Vassall A, van Kampen S, Sohn HS, et al. Rapid Diagnosis of Tuberculosis with the Xpert MTB/RIF Assay in high Burden Countries: a Cost-effectiveness Analysis. Public Library of Science. 2011;8(11):1001- 1120

22. Mustapha G, Jumoke O, Nwadike P, et al. Assessment of Gene-Xpert $\mathrm{MTB} / \mathrm{RIF}$ programme implementation and the challenges for enhanced tuberculosis diagnosis in Nigeria. J ubercule Lung Disease and HIV/ AIDS. 2015;2(1):324-239.

23. Oluwaseun E, Adeniyi BH, Olatunbosun OO, et al. Evaluation of the analytical performance of Xpert MTB/RIF Assay in the diagnosis of tuberculosis among HIV seropositive and seronegative patients in Abeokuta, Southwestern, Nigeria. Amer J Res Comm. 2015;3(10):1-14.

24. Zeka AN, Tasbakan S, Cavusoglu C. Evaluation of the genexpert MTB/RIF assay for rapid diagnosis of tuberculosis and detection of rifampicin resistance in pulmonary and extrapulmonary specimens. $J$ Clin Microbiol. 2011;49(12):4138-4141.

25. Ndubuisi NO, Azuonye OR, Victor NO. Diagnostic Accuracy of Xpert MTB/RIF Assay in Diagnosis of Pulmonary Tuberculosis. J. Infectious Disease and Treatment. 2016;2:23-35.

26. Hosne J, Sanya TJ, Zakir HH, et al. . Diagnostic evaluation of genexpert MTB/RIF assay for the detection of Rifampicin-Resistant mycobacterium tuberculosis among tuberculosis patients in Bangladesh. J Tuberculosis Res. 2016;4(1):55-60.

27. Haider AA, Abed AD, Humaira AR, et al. The Diagnostic Performance of a Single GeneXpert MTB/RIF Assay in an Intensified Tuberculosis Case Finding Survey among HIV-infected prisoners in Malaysia. Public Library of Science. 2013;8(9):1-10.

28. Azuonwu O, Nnenna I, Uwuma OE. Evaluation of haematological profile of geriatric subjects in port Harcourt metropolis of Niger Delta of Nigeria. J Clin Lab Med. 2017;2(1):111.

29. Azuonwu O, Nnenna I, Douglass AS, et al. Consequences of Haemolytic Disease of the Fetus and Newborn (HDFN) and the clinical significance of antibody screening in prenatal diagnosis: A Study of multigravidal and primigravidal women in Port Harcourt, Niger Delta. J Clin Lab Med. 2016;1(1):106. 